\title{
Pouvoir, autorité, légitimité
}

En marge d'un livre récent d'Alain Renaut

Jacques Coenen-Huther

\section{(2) OpenEdition}

Journals

Édition électronique

URL : http://journals.openedition.org/ress/471

DOI : $10.4000 /$ ress.471

ISSN : 1663-4446

Éditeur

Librairie Droz

Édition imprimée

Date de publication : 1 février 2005

Pagination : 135-145

ISBN : 2-600-00958-2

ISSN : 0048-8046

Référence électronique

Jacques Coenen-Huther, «Pouvoir, autorité, légitimité », Revue européenne des sciences sociales [En

ligne], XLIII-131 | 2005, mis en ligne le 12 novembre 2009, consulté le 19 avril 2019. URL : http://

journals.openedition.org/ress/471; DOI : 10.4000/ress.471 


\section{POUVOIR, AUTORITÉ, LÉGITIMITÉ En marge d'un livre récent d'Alain Renaut}

Dans un essai récent, La fin de l'autorité (2004), Alain Renaut poursuit une réflexion amorcée de longue date sur les choix de valeurs qui s'imposent aux sociétés démocratiques. Il reprend l'examen critique des problèmes que rencontrent l'action éducative et la relation adultes-enfants, déjà étudiés en détail dans La libération des enfants (2002). Ici, Renaut élargit considérablement le débat en situant ces problèmes dans un contexte de fragilisation généralisée de toutes les formes de pouvoir plus ou moins institutionnalisées. Partout où s'exerce un pouvoir, celui-ci est ébranlé par une crise de l'autorité qui est en fait une crise de légitimité. On doit savoir gré à l'auteur d'avoir montré l'ampleur d'un phénomène beaucoup plus important et plus complexe que ne le suggère telle ou telle de ses manifestations sectorielles. On appréciera également la référence dépourvue d'ambiguïté à la modernité. La remise en question de pouvoirs longtemps tenus pour inébranlables s'inscrit, parfois tardivement, dans la logique même de la modernité qui tend à dévaloriser les formes anciennes de domination. Point n'est besoin de faire intervenir à ce propos une théorie de la postmodernité. L'analyse de Renaut confère une actualité renouvelée à une opinion émise par Habermas il y a deux décennies, selon laquelle la modernité reste un projet inachevé (1985), même si les réaménagements institutionnels dont l'ouvrage offre la perspective sont qualifiés assez gratuitement de «postmodernes» (Renaut, 2004, pp. 128 et ss). Toutefois, si les réflexions qui nous sont ainsi proposées sont extrêmement stimulantes et méritent incontestablement un examen approfondi, elles n'en appellent pas moins remarques et réserves d'un point de vue sociologique.

\section{L'AUTORITÉ ET SES VARIANTES}

L'autorité est généralement considérée par les sociologues ${ }^{1}$ comme un pouvoir légitime: un pouvoir qui n'a donc besoin que d'un minimum de coercition pour se faire respecter et obéir. «On parle de l'autorité d'une personne, d'une institution, d'un message», écrivent Boudon et Bourricaud, «pour signifier qu'on leur fait confiance, qu'on accueille leur avis, leur suggestion ou leur injonction, avec respect, faveur, ou du moins sans hostilité ni résistance, et qu'on est disposé à y déférer» (1982, p. 24). L'autorité ne peut donc être considérée purement et

Mais pas seulement par les sociologues. Que l'on consulte à ce sujet le Dictionnaire encyclopédique de théorie et de sociologie du droit dirigé par André-Jean Arnaud (1993, pp. 51-53) ou le Dictionnaire de philosophie de Christian Godin (2004, p. 130). 
simplement comme un attribut du pouvoir car il s'agit d'un concept relationnel. Il ne peut être question d'autorité que dans le cadre d'une relation entre les détenteurs d'un pouvoir et ceux qui lui sont soumis. Il y a autorité quand un pouvoir bénéficie d'un capital de confiance et quand les individus sur qui le pouvoir s'exerce lui conservent leur confiance. Ce pouvoir est alors perçu comme légitime et il acquiert l'autorité de ce qui échappe à la contestation. C'est d'ailleurs bien dans cette optique que se place Alain Renaut puisqu'il se réfère aux fondements weberiens de la légitimité en y voyant des formes de «relations d'autorité»

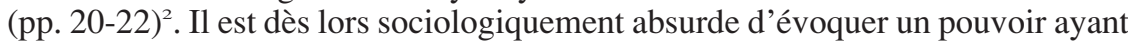
«recours à l'autorité» quand «il a besoin d'un surpouvoir» afin «d'obtenir l'obéissance» (p. 44). Le «surpouvoir», si surpouvoir il y a, est le résultat de l'exercice du pouvoir dans un climat de confiance et non la conséquence de la recherche délibérée d'un «surcroît de justification». Qu'une telle recherche en vienne à se manifester est au contraire l'indice que l'autorité s'est érodée au point de ne plus laisser subsister qu'un pouvoir mal assuré.

L'autorité, comme pouvoir légitimé, s'est appuyée sur la force de la tradition, sur l'attrait persuasif de dirigeants charismatiques ou, plus tardivement, sur les procédures et les garanties qu'offre l'ordre légal-bureaucratique. Il ne peut donc être question de «l'autorité» comme d'une denrée «énigmatique» qui viendrait renforcer le pouvoir. Il existe divers types d'autorité qui sont les produits émergents de formes différentes d'exercice du pouvoir. Celui-ci devient autorité quand il lui est possible de se fonder sur l'acceptation générale d'une argumentation légitimatrice implicite ou explicite. La «formule politique» selon Gaetano Mosca en offre un exemple ${ }^{3}$. On entend par là, écrit cet auteur, le fait que «la classe dirigeante justifie son pouvoir en le fondant sur une croyance ou sur un sentiment qui, à cette époque ou dans un peuple déterminé, sont généralement acceptés » (1955, p. 321). Pour être efficace, poursuit Mosca, la formule politique «doit correspondre à la conception du monde qui est, à un certain moment, celle du peuple considéré » (ibid., p. 322). Ainsi, l'autorité est le fruit de l'adhésion sans réserve à un discours légitimateur. Cette adhésion, tout comme son refus, n'implique pas nécessairement le contexte d'une société démocratique mais elle ne l'exclut pas non plus. Plus l'adhésion est forte, moins elle fait appel à la raison et plus l'autorité va de soi. La nature et le degré de l'adhésion sont des variables qui mettent en cause tant les modalités de l'exercice du pouvoir que les caractéristiques des individus sur lesquels il s'exerce. Il y a donc une autorité de type autoritaire tout comme une autorité de type démocratique, une autorité à caractère bureaucratique, fondée sur le respect de procédures, tout comme une autorité à caractère professionnel, fondée sur les qualifications et la compétence, une autorité appuyée sur une hiérarchie tout comme une autorité à caractère plus directement relationnel. Il est dangereux de ramener ces diverses figures de l'autorité à une catégorie unique qui serait en voie de disparition (p. 82). Certains types d'autorité sont plus vulnérables que d'autres et il n'est pas du tout certain, comme on le verra plus loin, que cet élément de vulnérabilité revête la même signification profonde dans

\footnotetext{
2 Dans la suite du texte, quand des numéros de pages sont indiqués sans référence d'auteur ou de date, c'est de La fin de l'autorité qu'il s'agit.

3 Je me permets de renvoyer sur ce point à ma Sociologie des élites (2004, pp. 48-51)
} 
tous les cas envisagés par Renaut: pouvoir politique, pouvoir pédagogique, pouvoir judiciaire, pouvoir médical. En bref, pour anticiper sur des développements ultérieurs, le pouvoir politique fait l'objet d'une crise de confiance qu'on peut qualifier de crise de légitimité, le pouvoir pédagogique est ébranlé par la montée du relativisme cognitif et moral, le pouvoir judiciaire est confronté à l'érosion de la responsabilité personnelle et le pouvoir médical est en butte à des phénomènes de frustration relative.

\section{L'ÉROSION DE L'AUTORITÉ}

Mais, nous dit-on, les tendances qui poussent à l'érosion plus ou moins rapide de l'autorité sous toutes ses formes sont non seulement de portée très générale mais elles sont irrésistibles dans la mesure où elles s'appuient sur des acquis irréversibles de la conscience démocratique (p. 29). Pour étayer cette thèse, Alain Renaut opère un rapprochement entre les «éléments non renégociables » de notre rapport au monde matériel et les convictions qui structurent notre conscience en matière de valeurs (pp. 29-30). Les «grandes découvertes» de la science nous ont fourni une série d'évidences qu'il n'est plus possible de remettre sérieusement en question: une résurgence du géocentrisme, par exemple, est devenue impensable. De même peut-il être question de «grandes créations axiologiques et normatives » sur lesquelles on ne saurait revenir. Des a priori philosophiques et moraux nous conduisent à traiter tout être humain «comme un semblable et comme un égal» (pp. 30-32). Le parallèle ainsi établi est séduisant mais il est discutable. Il est douteux que les principes gouvernant les rapports entre les humains se soient affirmés avec la même force que les composantes élémentaires de notre perception du monde. Certes, de nos jours, plus personne ne s'aviserait de prétendre que la terre est plate ou qu'elle est au centre de l'univers. Mais oserait-on affirmer que les conceptions prémodernes fondées sur le caractère naturel des hiérarchies et sur l'altérité fondamentale des groupes sociaux, des sexes, des classes d'âge, aient été irrévocablement balayées par les valeurs de la modernité? Les tragédies qui ont marqué le $\mathrm{XX}^{\mathrm{e}}$ siècle tout comme les crispations identitaires contemporaines permettent d'en douter.

Il n'en reste pas moins qu'on peut observer une tendance assez générale au réaménagement contractuel des relations de pouvoir. Pour tout ce qui concerne l'espace public, une telle contractualisation, soulignons-le, constitue en soi un élément de légitimation même s'il ne suffit plus à résorber tout déficit de légitimité. En fait, ainsi que l'observe Renaut, la légitimité de principe conférée par l'élection semble ne plus suffire à s'assurer la confiance des citoyens (p. 90). Ce sont deux «figures emblématiques» de la modernité politique - l'homme politique comme expert et l'homme politique comme leader - qui sont confrontées de nos jours à l'érosion de la légitimité du pouvoir, la politique comme expertise cherchant à se prévaloir de l' «autorité du savoir» et la politique comme leadership visant à se réserver le «moment de la décision» (pp. 101 et ss). Ces deux perspectives ne peuvent conduire logiquement qu'à «l'absolutisme des compétences» ou à «l'absolutisme de la décision». Dans l'un et l'autre cas, estime Renaut, le pouvoir revient à une élite et le modèle démocratique «perd toute véritable légitimité»(p. 118). Est-ce bien une incompatibilité de principe entre 
gouvernement des élites et régime démocratique qui est en cause? La démocratie ne peut-elle pas - ne doit-elle pas - se concrétiser au contraire dans des techniques institutionnelles qui assurent nécessairement l'émergence d'élites? C'est alors le critère de «lutte concurrentielle portant sur les votes du peuple» (Schumpeter, 1942, 1951, p. 403) qui permet de faire coexister élites et démocratie ${ }^{4}$ et l'on peut raisonnablement qualifier de démocratiques les régimes organisés de façon à permettre la concurrence pacifique des élites en vue de l'exercice du pouvoir. On rétorquera peut-être que c'est précisément une telle conception minimaliste de la démocratie qui est devenue problématique et qui ébranle la légitimité du pouvoir. Mais il est très vraisemblable que le «déficit de légitimité » ne soit pas dû aux mécanismes institutionnels eux-mêmes et qu'il ait des causes bien plus profondes, à rechercher dans la crise du lien social déjà pressentie par Tocqueville et dans la logique de l'individualisme moderne qui va bien au-delà de l'affirmation de l'autonomie du sujet. Comme le fit observer Dahrendorf, pour que la légalité se transforme en légitimité, il faut que les normes ne s'appuient pas seulement sur des sanctions mais aussi sur des liens (1984, p. 140; souligné par JCH). C'est la notion même de «souveraineté du peuple» qui s'efface des consciences ${ }^{5}$. Pour Rousseau, la vie politique de la Cité reposait sur un dédoublement conceptuel de l'individu qui a renoncé à la «liberté naturelle» de l'état de nature pour adhérer au pacte social. Comme membre du «corps politique», c'est-à-dire comme membre d'un tout, cet individu participe de la toute-puissance du peuple souverain et contribue à établir la «volonté générale». En revanche, comme particulier, ce même individu est totalement soumis à la communauté. Rousseau y insiste fortement; il écrit: «s'il restait quelques droits aux particuliers... chacun étant en quelque point son propre juge prétendrait bientôt l'être en tous...». Le pacte social renferme donc «tacitement» l'engagement «que quiconque refusera d'obéir à la volonté générale y sera contraint par tout le corps» car on ne saurait vouloir jouir « des droits du citoyen sans vouloir remplir les devoirs du sujet» (Rousseau, 1762, 1966, pp. 50-55). Dans la logique de l'individualisme exacerbé, tout se passe comme si la dualité imaginée par Rousseau cessait de rendre compte adéquatement de la vie publique parce que cette dualité est fondée sur un équilibre précaire entre la liberté des Anciens et la liberté des Modernes. Le «particulier » prétend en effet «être son propre juge» en toute chose et le collectif se sent impuissant à le ramener à une conception plus raisonnable des exigences du pacte social. Le particulier tente de faire prévaloir sa volonté et le corps politique est de moins en moins à même de le rappeler à l'ordre. Cette relative impuissance face aux forces centrifuges n'est pas seulement politique; elle est aussi d'ordre intellectuel et moral car l'individualisme contemporain véhicule un relativisme qui achève d'ébranler la légitimité des interventions de la puissance publique. Le politique «meneur de jeu » que Renaut appelle de ses vœux (p. 131) serait inévitablement conduit à s'en remettre aux instruments de persuasion de la compétence et du charisme car il n'y a pas, en matière politique, d'argument intrinsèquement «meilleur» (p. 132) dans

4 A ce sujet, je renvoie à nouveau à ma Sociologie des élites (2004, pp.58-62).

5 J'emprunte l'argumentation qui suit à la communication que j'ai présentée au $17^{\circ}$ congrès de 1'Association Internationale des Sociologues de Langue Française (Tours, juillet 2004) sous le titre Faut-il reconsidérer l' homo sociologicus? (à paraître). 
la mesure où toute décision est basée sur une combinaison inextricable de jugements de valeur et d'informations.

Même si l'analogie est tentante, il ne paraît guère possible d'envisager la mise en œuvre de la logique du contrat dans les mêmes termes, dans l'espace public et dans le domaine privé, entre adultes ou entre adultes et enfants, entre représentants de la loi et délinquants, entre les médecins et leurs patients. L'espace public est régi au moins en principe par des normes universalistes s'appliquant à des rapports sociaux spécifiques, dans un contexte de neutralité affective, entre individus traités en fonction de leurs capacités et de leurs actions davantage qu'en fonction de leurs caractéristiques d'appartenance. Au contraire, le domaine privé, et singulièrement le milieu familial, est dominé tout à fait légitimement par des normes particularistes portant sur des relations à caractère diffus, dans un climat chargé d'affectivité, entre individus appréciés pour leurs affiliations et leurs allégeances plutôt que pour leurs accomplissements ${ }^{6}$. Le complexe de normes universalistes liées à l'espace public se prête sans trop de difficultés logiques à une contractualisation plus ou moins poussée. Il y a, pourrait-on dire, affinité entre de telles normes et l'esprit de la contractualisation. En revanche, les normes particularistes liées au domaine privé définissent un espace social rebelle à toute réglementation, fût-elle d'esprit contractuel. Si la contractualisation appliquée à l'espace public peut, sous certaines conditions, conférer une légitimité nouvelle au pouvoir politique, il est loin d'en aller de même pour l'espace privé. La contractualisation confrontée au complexe de normes particularistes est avant tout source de désorganisation sociale. Un juridisme envahissant pénètre de nos jours un domaine où, selon la formule consacrée, «les lois se taisent», marquant ainsi une limite raisonnable aux interventions de la puissance publique. Affectant le lien familial, cette juridicisation n'est nullement porteuse d'une réorganisation contractuelle mais contribue au contraire à affaiblir l'autorité parentale, perturbant ainsi des relations de pouvoir nécessairement inégalitaires tout en intervenant de façon malencontreuse dans un domaine qui gagnerait à rester régi par des relations d'affinité.

\section{LA LOGIQUE DU CONTRAT ET LA RELATION ÉDUCATIVE}

L'idée que la logique du contrat puisse s'appliquer aux relations entre les éducateurs et les enfants qu'il s'agit d'éduquer repose entièrement sur l'a priori d'égalité et de similitude évoqué plus haut. Dans cette perspective, l'enfant, du fait de son incontestable appartenance à l'humanité, serait un sujet de droit au même titre que l'adulte. Si l'on n'a aucune difficulté à admettre qu'il le soit pour ce qui concerne les «droits-protection» - ce que Renaut appelait les «droitscréance» dans La libération des enfants - il est impossible de se rallier à ce point de vue pour ce qui est des «droits-libertés». A tous les stades de son développement, l'enfant doit bénéficier, non seulement de tous les «droits-créance » qu’on

On l'aura compris, je m'inspire ici des pattern-variables, les variables de configuration parsoniennes (Parsons, 1951, pp. 66-67) 
accorde de nos jours à un être humain, mais également de la sollicitude particulière que requiert sa faiblesse et son immaturité. L'enfant est à certains égards un sujet de droit mais à d'autres égards un objet de droit. Il n'est certainement pas un sujet de droit à part entière. Si donc la contractualisation doit gagner du terrain dans les tâches éducatives, il ne peut s'agir d'un contrat entre adultes et enfants mais bien d'un contrat entre différentes instances et différentes catégories d'acteurs participant à l'effort éducatif. Que les options en matière éducative soient discutées et négociées entre adultes concernés est bien conforme à l'esprit d'une société démocratique. En revanche, une action éducative qui se soumettrait potentiellement à la censure de ses bénéficiaires se mettrait, aujourd'hui comme autrefois, en contradiction avec elle-même. La relation éducative est nécessairement par définition, pourrait-on dire - une relation inégalitaire où l'un inculque à l'autre des usages, des savoirs et des savoir-faire qu'il juge souhaitable de lui inculquer. Ce processus de transmission/inculcation peut être mis au service de la démocratie; il ne participe pas de la démocratie. Ceci n'apparaît comme une incongruité qu'à ceux qui entendent pousser jusqu'à l'absurde le «régime de la similitude» de tous les êtres humains (p. 149) et à l'étendre à l'enfant. Celui-ci, n'hésitons pas à l'affirmer, doit être exclu «du statut de semblable» (ibid.) si l'on veut échapper aux apories de la relation éducative confrontée à la montée de l'égalitarisme et de l'éthique de la discussion. Pour l'adulte qui l'élève, l'enfant n'est pas un semblable: il est un futur semblable, un semblable en puissance qu'on se fait un devoir d'amener peu à peu à la pleine maturité. Hannah Arendt avait raison d'écrire que la «libération des enfants » est un leurre, qu' « affranchi de l'autorité des adultes», les enfants se retrouvent «livrés à la tyrannie de leur groupe » qui n'offre de loin pas les mêmes possibilités de discussion que le «monde des adultes » (Arendt, 1954, 1972, p. 233). Depuis l'essai prémonitoire d'Arendt, de nombreux psychologues ont mis l'accent sur ce phénomène paradoxal de libération-aliénation. Et l'idée qu'une autorité plus ferme, telle qu'on la pratiquait il y a un demi-siècle, était finalement bénéfique à l'enfant, n'est pas fondée uniquement sur «d'extravagantes idéalisations » du passé (Renaut, 2004, p. 144). La nostalgie de rôles plus différenciés, de limitations plus strictes et d'injonctions plus fortes n'est pas seulement le fait d'esprits chagrins ou rétrogrades. Ici encore, les psychologues de l'enfance font état d'une sorte de vertige provoqué par l'affaiblissement de repères normatifs autrefois plus nets. A ce propos, la montée du conformisme de groupe et de la délinquance juvénile, déjà signalées par Hannah Arendt (1954, 1972, pp. 233-234), nous invite à la réflexion. Les situations à la Dickens évoquées par Renaut (pp. 158 et ss) nous font aujourd'hui frémir et plus personne ne songerait à y revenir. Qu'à cet égard l'idée de «l'enfant porteur de droits » constitue un barrage efficace contre certaines horreurs du passé n'est pas niable. Mais la confusion entre droits-protection et droits-libertés ne constitue pas un progrès supplémentaire et ne profite certainement pas à l'enfance.

La relation adulte-enfant ne peut s'inscrire purement et simplement dans le «régime du même» (p. 146) et il est exagéré de parler de «rupture radicale » entre l'éducation et le dressage (p. 195). Le bébé qui vient de naître n'est guère plus qu'une promesse d'humain. Le développement d'un jeune enfant peut être interprété comme un processus de construction de la nature humaine. Diverses maturations organiques qui, chez de nombreux animaux, ont lieu avant la naissance, se déroulent chez l'enfant dans la période postnatale. Les mammifères supérieurs 
achèvent dans l'utérus la formation de leur corps ainsi que l'essentiel de la maturation de leur système nerveux. A la naissance, le mammifère supérieur est l'image de l'adulte pour ce qui est de la forme, du comportement et de la motricité (Portmann, 1951, p. 71). Il n'en va pas de même pour les humains. Lorsqu'on fait le rapport entre le développement cérébral et la durée de gravidité, "on constate que la gravidité devrait être bien plus longue pour produire un petit être conforme aux règles de développement des primates» (ibid.) Ce n'est que pendant la première année extra-utérine que se complète la maturation neuro-musculaire de l'enfant (ibid., p. 72). C'est donc l'environnement social de l'enfant - les adultes qui prennent soin de lui - qui le met en mesure d'accéder pleinement à l'humanité. En dépit des fables d'enfants-loups, le bébé humain n'est pas à même d'y accéder par lui-même. Pendant une période relativement longue, le processus de socialisation s'apparente bel et bien à une forme de dressage. Ainsi en est-il par exemple de l'apprentissage des règles élémentaires de propreté corporelle et d'hygiène ainsi que de la soumission aux rythmes alimentaires. A un stade ultérieur, la transmission de normes de politesse et de civilité se fonde davantage sur des procédés d'inculcation que sur des pratiques éducatives faisant appel à la raison, cela d'autant plus qu'il s'agit de règles de conduite ayant un caractère en grande partie conventionnel et qui sont donc particulièrement vulnérables à la contestation. C'est très progressivement que l'argumentation rationnelle peut se substituer à un quasi-dressage.

\section{LES DILEMMES DE LA PRATIQUE PÉNALE}

Dégagé de toute référence à quelque transcendance, le droit de punir ne peut se fonder que sur la rupture du pacte social: on sera d'accord en cela avec Renaut. Observons cependant que la contractualisation de la pénalité est aussi problématique que celle du pouvoir pédagogique. Les délinquants qui se sont mis en marge de la légalité ne peuvent être davantage partie prenante à un débat sur les options de la politique judiciaire que les enfants ne le sont à un débat sur les orientations de l'action éducative. Une refondation contractualiste de la pratique pénale, note Renaut, favorise une conception «utilitariste» de la peine. Celle-ci se décide alors en fonction d'impératifs de «paix civile» (p. 203). L'objection kantienne reste cependant valable et il subsiste de bons arguments pour défendre l'idée que la peine constitue un but en soi et qu'elle doit intervenir dès lors qu'un crime a été commis, et pour cette simple raison qu'il a été commis. Un ordre social s'appuyant sur une échelle de valeurs ferme ne peut laisser des considérations d'opportunité présider à la détermination ou à l'application de la peine. Les hésitations fréquentes du pouvoir judiciaire et du pouvoir législatif, partagés entre la conception utilitariste et la conception autonomiste de la pénalité, mais penchant de plus en plus vers la première, ne peuvent qu'affaiblir le respect de la norme dans la population. Fichte avait sans doute raison de mettre l'accent sur la différence entre le droit et la morale mais cette différence n'exclut évidemment pas les interactions. Les règles de droit constituent somme toute un ensemble assez disparate de normes ayant des statuts très divers. Certaines d'entre elles apparaissent comme l'application de principes moraux très forts. D'autres se présentent au contraire comme des compromis entre des conceptions morales antagonistes. D'autres 
enfin ne semblent avoir aucun rapport direct avec une morale quelconque. Ce rapport peut toutefois s'établir de manière indirecte, par le truchement du devoir civique de respect de règles purement conventionnelles. Il faut bien voir aussi que les options prises en matière de droit ont des retombées en matière de moralité publique. A ce propos, il est un aspect de l'action pénale dont l'importance, de nos jours, tend à être minimisé, ce qui constitue certainement un effet pervers de la conception utilitariste. Il s'agit de l'attitude à adopter à l'égard des victimes de la délinquance de tous niveaux, depuis les délits mineurs jusqu'à la grande criminalité. Pourtant, l'émotion ou la souffrance des victimes mérite d'être prise en considération, tant dans une optique utilitariste - celle de la paix civile - que dans une optique autonomiste: celle de la peine comme fin en soi. La douleur, l'indignation, la dignité blessée alimentent l'appel au châtiment ainsi qu'un désir de vengeance qui devrait rencontrer un peu plus de compréhension qu'il n'en suscite à l'heure actuelle. Il n'y a pas de raison, en tout cas, de le traiter avec dédain, comme une résurgence de temps anciens.

Tout l'édifice moderne de l'action judiciaire repose sur un postulat de liberté humaine et de responsabilité personnelle. Renaut a certainement raison de souligner les conséquences regrettables de la «psycho-sociologisation» du crime. La mise en évidence unilatérale de facteurs psychiques ou sociaux qui exonèrent au moins partiellement le criminel de sa responsabilité ne facilite guère la réhabilitation de ce dernier. Il devient extrêmement difficile de l'amener à se penser comme le «sujet» de ses actes (pp. 224-225). A ce propos, nombreux sont les sociologues qui devraient s'interroger sur la portée de leurs analyses et se demander s'ils n'ont pas encouragé un angélisme malavisé 7 . Mais tout aménagement contractualiste de la pratique de la pénalité se heurte très rapidement aux incertitudes qui compliquent la relation adulte-enfant. Si la modernité a lié l'action de punir à la notion de responsabilité personnelle - et si, pour cette raison, seul l'être humain, capable d'actions intentionnelles, peut faire l'objet de peines - on retombe dans des contradictions difficiles à surmonter lorsque les délinquants sont des enfants. Veut-on, dans une optique de protection sociale, faire bénéficier l'enfant d'une mansuétude particulière et le soumettre à une juridiction spéciale, il s'impose de prendre appui sur un postulat de responsabilité inexistante ou atténuée. Veut-on au contraire, dans l'esprit de la Convention sur les droits de l'enfant de 1989, se placer dans la logique de «l'enfant-citoyen », il devient alors très difficile de maintenir le principe de l'irresponsabilité pénale des mineurs qui constitue pourtant un élément majeur du dispositif de protection de l'enfance.

\section{L'ÉBRANLEMENT DU POUVOIR MÉDICAL}

Selon l'interprétation qu'en offre Alain Renaut, l'ébranlement du pouvoir médical correspond au fond à un phénomène bien connu des sociologues. On l'a appelé «l'effet Tocqueville» ou, plus généralement, le processus de frustration

Comme je l'ai déjà suggéré dans une publication récente (2003, p. 171). 
relative $^{8}$. Les avantages acquis - en l'occurrence, les bienfaits de la médecine classique - sont tenus pour allant de soi et cessent d'être une source de satisfaction. Ce qui ne peut être obtenu - et ne pourra jamais l'être - c'est l'état de bienêtre généralisé promis par l'absurde définition de la santé de l'OMS. Comme celle-ci n'en est pas moins en phase avec l'air du temps, des aspirations impossibles à satisfaire se sont répandues dans la population qui ne peut qu'être déçue par les médecins. Soit dit en passant, il est étonnant que Renaut ne se soit pas montré aussi dur avec la déclaration des droits de l'enfant de 1989 qu'avec la définition de la santé de l'OMS. Car si cette définition a bien un côté «comique», style «Knock» (pp. 244-245), la déclaration de 1989 ne le lui cède en rien lorsqu'elle aligne de prétendus droits-libertés qui ne peuvent qu'entrer en conflit avec les droits-protection.

Le médecin, porté par un niveau d'aspirations qui s'est considérablement élevé en quelques décennies, voit son rôle professionnel se transformer. L'expert en matière de santé est devenu bon gré mal gré un «psychothérapeute» général (p. 247), rôle pour lequel il est mal armé et dans l'exercice duquel ses compétences sont beaucoup plus faciles à contester. Cette évolution était déjà contenue en germe il y a un demi-siècle, dans ce que Parsons qualifiait de «fonction latente» de psychothérapie de la médecine (Parsons, 1951, p. 478). Comme le fait observer Renaut, l'élargissement considérable du champ d'intervention de la médecine expose le pouvoir médical à un risque élevé de délégitimation dans la mesure où les déceptions ne peuvent que se multiplier. L'anecdote du «pantalon baissé» (Renaut, 2004, p. 254) - à savoir le monopole de l'accès à l'intimité corporelle de l'individu malade - garde cependant toute sa pertinence et impose des limites à l'assaut du pouvoir médical par la «culture de l'égalité». La situation du malade est en effet une situation sociale spécifique que la contractualisation ambiante n'a que marginalement transformée. Le rôle de l'individu malade, selon l'analyse classique de Parsons, est défini par des attentes plus ou moins institutionnalisées, assorties de sanctions sociales, formelles ou diffuses (1951, p. 436). Tout d'abord, le malade est exempté des responsabilités associées normalement à un rôle social. Cette exemption est à la fois un droit et un devoir. Ensuite, il est admis que le malade ne puisse se rétablir par un acte de pure volonté. En ce sens, le malade est également exempté de responsabilités. La maladie est perçue comme une «condition» qui doit être soignée et qui requiert de l'aide. En troisième lieu, le malade doit avoir le désir de guérir. On attend de lui qu'il définisse sa situation comme non désirable. La suspension des responsabilités n'est légitimée que si cette condition est remplie. Enfin, le malade est censé rechercher l'aide de quelqu'un de compétent. On attend le plus souvent de lui qu'il s'adresse à un médecin et qu'il coopère avec lui (ibid., p. 347). En dépit des exhortations à la responsabilisation des patients et de la judiciarisation qui a envahi également le monde de la santé, la condition sociale du malade est faite d'une atténuation des responsabilités et d'une incontestable pression à la conformité qui va dans le sens d'une soumission au pouvoir médical, même s'il s'agit plus qu'autrefois d'une soumission assortie de récriminations. Des différents types de pouvoir passés en revue, le pouvoir médical, comme le note Renaut, ne s'en sort pas trop mal.

\footnotetext{
8 Voir à ce sujet mon Tocqueville (1997; pp. 96 et ss).
} 
Remarquons toutefois que ce n'est pas dans la lutte contre le «mal-être »que le médecin se voit placé sur la défensive, mais bien dans la part de sa pratique qui continue à relever du modèle de la médecine scientifique. C'est le droit à l'erreur qu'on lui reconnaît de plus en plus difficilement ${ }^{9}$, ce qui offre aux juristes, ici comme dans la relation à l'enfance, des possibilités inédites d'accroissement de leur propre pouvoir.

\section{POUR CONCLURE}

Quel que soit son domaine d'application, l'argumentation d'Alain Renaut est sous-tendue par un sentiment d'irréversibilité. C'est pourquoi, en dépit d'un sens des nuances qui commande le respect, il nous oriente systématiquement vers des perspectives «progressives » plutôt que vers des perspectives « réactives », partant de l'idée que tout retour à des formes de pouvoir plus solide est exclu. Certes, dans le cours de l'histoire, il est des évolutions à long terme d'une telle force qu'on voit mal comment elles auraient pu être infléchies. Ainsi en est-il de la tendance à l'égalisation progressive des conditions mise en évidence par Tocqueville. Pourtant, dès qu'on s'écarte des processus de changement social de très longue durée, la liberté humaine s'accommode mieux d'une conception cyclique de l'histoire que d'une conception linéaire. La tendance à la contractualisation/délégitimation signalée par Renaut suscite-t-elle vraiment des processus irréversibles dans tous les domaines? S'agissant de la relation éducative, rien n'est moins sûr. Dans un premier temps, les « utopies pédagogiques des années 1960 et 1970 » ont déployé leurs «effets dévastateurs» (Renaut, p. 228) de façon apparemment irrésistible. Ces effets se sont fait sentir de proche en proche jusqu'au niveau universitaire et ont semblé donner lieu à une démission éducative généralisée. Quelques décennies plus tard, des voix de plus en plus nombreuses se font entendre pour appeler ouvertement à un renversement de tendance. Il fallait tout d'abord que quelques individus aient le courage de crier dans le désert et d'affronter la réprobation de leurs concitoyens moins lucides. Une prise de conscience plus large en résulte finalement. La liberté humaine n'a pas dit son dernier mot.

\section{RÉFÉRENCES BIBLIOGRAPHIQUES}

Arendt, Hannah, Between Past and Future, 1954, tr. fr. La crise de la culture, Paris, Gallimard, Folio/Essais, 1972.

Arnaud, André-Jean, Ed., Dictionnaire encyclopédique de théorie et de sociologie du droit, Paris, LGDJ, deuxième édition, 1993.

Boudon, Raymond et François Bourricaud, Dictionnaire critique de la pensée sociologique, Paris, PUF, 1982.

Coenen-Huther, Jacques, Tocqueville, Paris, PUF, Coll. «Que sais-je?», 1997.

\footnotetext{
Signalons à ce propos qu'un jeune sociologue de l'Université de Genève, Raphaël Hammer, dans une thèse de doctorat consacrée aux représentations de la médecine, relève une tendance, non exclusive mais persistante, à considérer le médecin comme un professionnel dont on attend qu'il s'en tienne strictement à son rôle.
} 
Coenen-Huther, Jacques, «Esprit sociologique et relativisme moral», in Revue Européenne des Sciences Sociales, Tome XLI, No 126, 2003, pp. 169-180

Coenen-Huther, Jacques, Sociologie des élites, Paris, Armand Colin, Coll. Cursus, 2004.

Dahrendorf, Ralf, Reisen nach innen und außen. Aspekte der Zeit, Stuttgart, Deutsche Verlags-Anstalt, 1984.

Godin, Christian, Dictionnaire de philosophie, Paris, Fayard / Editions du temps, 2004.

Habermas, Jürgen, «Modernity - An Incomplete Project», in: Hal Foster, Ed., Postmodern Culture, Londres, Pluto Press, 1985.

Mosca, Gaetano, Lezioni di storia delle istituzioni e delle dottrine politiche, 1933, 1937, tr. fr. par Gaston Bouthoul: Histoire des doctrines politiques depuis l'Antiquité jusqu'à nos jours, Paris, Payot, 1937, nouvelle édition, 1955.

Parsons, Talcott, The Social System, Londres, Routledge and Kegan Paul, 1951.

Portmann, Adolphe, «Les bases biologiques d'un nouvel humanisme», in: E. Guyénot, Le problème de la vie, Neuchâtel, La Baconnière, 1951.

Renaut, Alain, La libération des enfants, Paris, Calmann-Lévy / Bayard, 2002.

Renaut, Alain, La fin de l'autorité, Paris, Flammarion, 2004.

Rousseau, Jean-Jacques, Du contrat social, 1762, Paris, Garnier/Flammarion, 1966.

Schumpeter, Joseph, Capitalism, Socialism and Democracy, 1942, tr. fr. Capitalisme, socialisme et démocratie, Paris, Payot, 1951. 